\title{
Generalization of $(0,2,5)$ Lacunary interpolation by sixtic splines on uniform meshes
}

\author{
Ridha G.karem \\ Department of Mathematical Science, University of Sulaimani
}

\begin{abstract}
:
Spline functions are the best tool of polynomials used as the basic means of approximation theory in nearly all areas of numerical analysis. Also in the problem of interpolation by $\mathrm{g}$-spline construction of spline, existences, uniqueness and error bounds needed.

In this study, we generalized $(0,2,5)$ Lacunary interpolation by sixtic spline on uniform meshes. The results obtained, the existence uniqueness and error bounds for generalize $(0,2,5)$ Lacunary interpolation by sixtic spline. These generalize are preferable to interpolation by sixtic spline to the use $(0,2,5)$.
\end{abstract}

\section{Introduction:}

Spline functions are the best tool of polynomials used as the basic means of approximation theory in nearly all areas of numerical analysis. One uses polynomial for approximation because they can be evaluated, differentiated and integrated easily and in finitely many steps using the basic arithmetic operations of addition, subtraction, division and multiplication. Spline functions constitute a relativity new subject in analysis. During the past twentieth both the theories of splines and experiences with their use in numerical analysis have under gone a considerable degree of development. The following works deal to various degree with the theory and application of splines, (Ahlberg et al., 1967). In addition to the papers mentioned above dealing with best interpolation or approximation by splines, There were also a few papers that deal with constructive properties of space of spline interpolation (Kanth et al., 2006; Khan and Aziz, 2003; Siddiqi et al., 2007). In this study we studied the generalization of one type of Lacunary interpolation by sixtic spline this type is $(0,2,5)$. Also in the future we can use the same idea for different Lacunary interpolation that means we can generalities for different cases in the subjected Lacunary interpolation by spline. We have Hermite interpolation if for each $i$, the order $\mathrm{j}$ of derivatives in (1) from unbroken Sequence. If some of the sequences are broken, we have Lacunary interpolation. The Lacunary interpolation problem, which we have investigated in this study, consists in finding the six degree spline $S(x)$, interpolating data given on the function value and fourth order in the interval $[0,1]$. Also, an extra initial condition is prescribed on the first derivative.

This study is organized as follows: First consider the spline function of degree six is presented which interpolates the Lacunary data $(0,2,5)$. Some theoretical results about existence, uniqueness and error bounds of the spline function of degree six are introduced and also convergence analysis is studied. To demonstrate the convergence of the prescribed Lacunary spline function. 


\subsection{Descriptions of the Method:}

In this section, We present for the first time according to our knowledge a six degree spline $(0,2,5)$ interpolation for one dimensional and given sufficiently smooth function $f(x)$, defined on $i=[0,1]$ and:

$$
P_{n}^{(j)}\left(x_{i}\right)=a_{i, j}, i=1,2, \ldots, n ; j=0,1,2, \ldots, n
$$

We have Hermite interpolation if for each $i$, the order $j$ of derivatives in (1) from unbroken Sequence. If some of the sequences are broken, we have Lacunary interpolation:

$$
\Delta_{n}: 0=x_{0}<x_{1}<\cdots<x_{n}=1
$$

Denote the uniform partition of $\mathrm{i}$ with knots: $x_{i}=i h \quad$ where $h=x_{i+1}-x_{i} \quad i=1,2, \ldots, n-1$

We define the class of spline function $S_{n, 5}^{2}$ where $S_{n, 5}^{2}$ denotes the class of all splines of degree six which belongs to $C^{2}[0,1]$, and $\mathrm{n}$ is the number of knots , as follow :

Any element $S_{\Delta}(x) \in S_{n, 6}^{2}$ if the following two conditions are satisfied:

(i) $S_{\Delta}(x) \in C^{2}[0,1]$

(ii) $S_{\Delta}(x)$ is a polynomial of degree six in each $\left[x_{i}, x_{i+1}\right], i=0,1, \ldots, n-1$

\section{Construction of the Lacunary sixtic Spline Function:}

If $S(x)$ is a polynomial of degree six on $[0,1]$, then we have

$$
\begin{aligned}
S(x)= & S_{\lambda}(0) A_{0}(t)+S_{\lambda}(\lambda) A_{1}(t)+S_{\lambda}(1) A_{2}(t)+S_{\lambda}^{\prime}(0) A_{3}(t)+S_{\lambda}^{\prime}(1) A_{4}(t)+ \\
& S_{\lambda}^{\prime \prime}(\lambda) A_{5}(t)+S_{\lambda}^{(5)}(\lambda) A_{6}(t),
\end{aligned}
$$

Where $\lambda \in(0,1)$

$$
\begin{gathered}
\mathrm{A}_{0}(\mathrm{t})=-\frac{\left(6 \lambda^{2}+3 \lambda-1\right)}{\lambda\left(15 \lambda^{4}-15 \lambda^{3}+4 \lambda^{2}\right)} \mathrm{t}^{6}+\frac{6\left(6 \lambda^{2}+3 \lambda-1\right)}{15 \lambda^{4}-15 \lambda^{3}+4 \lambda^{2}} \mathrm{t}^{5}-\frac{3\left(20 \lambda^{4}+20 \lambda^{3}-5 \lambda+1\right)}{\lambda\left(15 \lambda^{4}-15 \lambda^{3}+4 \lambda^{2}\right)} \mathrm{t}^{4} \\
+\frac{2\left(15 \lambda^{5}+45 \lambda^{4}+10 \lambda^{3}-15 \lambda^{2}+1\right)}{\lambda\left(15 \lambda^{4}-15 \lambda^{3}+4 \lambda^{2}\right)} \mathrm{t}^{3} \frac{3\left(15 \lambda^{4}+5 \lambda^{3}-6 \lambda+2\right)}{15 \lambda^{4}-15 \lambda^{3}+4 \lambda^{2}} \mathrm{t}^{2} \\
\quad-\frac{3\left(15 \lambda^{4}+5 \lambda^{3}-6 \lambda+2\right)}{15 \lambda^{4}-15 \lambda^{3}+4 \lambda^{2}} \mathrm{t}^{2}+1,
\end{gathered}
$$




$$
\begin{aligned}
& A_{1}(t)=\frac{\left(6 \lambda^{2}-6 \lambda+1\right)}{\lambda\left(-15 \lambda^{7}+60 \lambda^{6}-94 \lambda^{5}+72 \lambda^{4}-27 \lambda^{3}+4 \lambda^{2}\right)} t^{6}+\frac{6\left(6 \lambda^{2}-6 \lambda+1\right)}{-15 \lambda^{7}+60 \lambda^{6}-94 \lambda^{5}+72 \lambda^{4}-27 \lambda^{3}+4 \lambda^{2}} t^{5}- \\
& \frac{3\left(15 \lambda^{4}-18 \lambda^{2}+8 \lambda-1\right)}{\lambda\left(-15 \lambda^{7}+60 \lambda^{6}-94 \lambda^{5}+72 \lambda^{4}-27 \lambda^{3}+4 \lambda^{2}\right)} \mathrm{t}^{4}+\frac{2\left(45 \lambda^{4}-54 \lambda^{3}+12 \lambda^{2}+3 \lambda-1\right)}{\lambda\left(-15 \lambda^{7}+60 \lambda^{6}-94 \lambda^{5}+72 \lambda^{4}-27 \lambda^{3}+4 \lambda^{2}\right)} \mathrm{t}^{3}- \\
& \frac{3\left(15 \lambda^{3}-24 \lambda^{2}+12 \lambda-2\right)}{-15 \lambda^{7}+60 \lambda^{6}-94 \lambda^{5}+72 \lambda^{4}-27 \lambda^{3}+4 \lambda^{2}} t^{2} \\
& A_{2}(t)=\frac{\left(6 \lambda^{2}-15 \lambda+8\right)}{15 \lambda^{5}-60 \lambda^{4}+94 \lambda^{3}-72 \lambda^{2}+27 \lambda-4} t^{6}-\frac{6\left(6 \lambda^{3}-15 \lambda^{2}+8 \lambda\right)}{15 \lambda^{5}-60 \lambda^{4}+94 \lambda^{3}-72 \lambda^{2}+27 \lambda-4} t^{5}+ \\
& \frac{12\left(5 \lambda^{4}-10 \lambda^{3}+5 \lambda-1\right)}{15 \lambda^{5}-60 \lambda^{4}+94 \lambda^{3}-72 \lambda^{2}+27 \lambda-4} t^{4}+\frac{10\left(-3 \lambda^{5}+16 \lambda^{3}-15 \lambda^{2}+3 \lambda\right)}{15 \lambda^{5}-60 \lambda^{4}+94 \lambda^{3}-72 \lambda^{2}+27 \lambda-4} t^{3}- \\
& \frac{3\left(-15 \lambda^{5}+40 \lambda^{4}-30 \lambda^{3}+6 \lambda^{2}\right)}{15 \lambda^{5}-60 \lambda^{4}+94 \lambda^{3}-72 \lambda^{2}+27 \lambda-4} t^{2} \\
& A_{3}(t)=-\frac{(3 \lambda-1)}{\lambda\left(15 \lambda^{3}-15 \lambda^{2}+4 \lambda\right)} t^{6}+\frac{6(3 \lambda-1)}{15 \lambda^{3}-15 \lambda^{2}+4 \lambda} t^{5}-\frac{\left(30 \lambda^{3}+10 \lambda^{2}-15 \lambda+3\right)}{\lambda\left(15 \lambda^{3}-15 \lambda^{2}+4 \lambda\right)} t^{4}+ \\
& \frac{\left(15 \lambda^{4}+45 \lambda^{3}-30 \lambda^{2}+2\right)}{\lambda\left(15 \lambda^{3}-15 \lambda^{2}+4 \lambda\right)} \mathrm{t}^{3}-\frac{6\left(5 \lambda^{3}-3 \lambda+1\right)}{15 \lambda^{3}-15 \lambda^{2}+4 \lambda} \mathrm{t}^{2}+\mathrm{t} \\
& A_{4}(t)=-\frac{(3 \lambda-2)}{15 \lambda^{4}-45 \lambda^{3}+49 \lambda^{2}-23 \lambda+4} t^{6}-\frac{6\left(2 \lambda-3 \lambda^{2}\right)}{15 \lambda^{4}-45 \lambda^{3}+49 \lambda^{2}-23 \lambda+4} t^{5}+\frac{2\left(-15 \lambda^{3}+5 \lambda^{2}+5 \lambda-1\right)}{15 \lambda^{4}-45 \lambda^{3}+49 \lambda^{2}-23 \lambda+4} t^{4}+ \\
& \frac{5\left(3 \lambda^{4}+3 \lambda^{3}-5 \lambda^{2}+\lambda\right)}{15 \lambda^{4}-45 \lambda^{3}+49 \lambda^{2}-23 \lambda+4} t^{3}-\frac{3\left(5 \lambda^{4}-5 \lambda^{3}+\lambda^{2}\right)}{15 \lambda^{4}-45 \lambda^{3}+49 \lambda^{2}-23 \lambda+4} t^{2} \\
& A_{5}(t)=-\frac{1}{2 \lambda\left(15 \lambda^{3}-30 \lambda^{2}+19 \lambda-4\right)} t^{6}+\frac{3}{15 \lambda^{3}-30 \lambda^{2}+19 \lambda-4} t^{5}-\frac{\left(5 \lambda^{2}+10 \lambda-3\right)}{2 \lambda\left(15 \lambda^{3}-30 \lambda^{2}+19 \lambda-4\right)} t^{4}+ \\
& \frac{\left(5 \lambda^{2}+\lambda-1\right)}{\lambda\left(15 \lambda^{3}-30 \lambda^{2}+19 \lambda-4\right)} t^{3}-\frac{(5 \lambda-2)}{30 \lambda^{3}-60 \lambda^{2}+38 \lambda-8} t^{2} \\
& A_{6}(t)=\frac{(2 \lambda-1)}{900 \lambda^{2}-900 \lambda+240} t^{6}-\frac{\left(9 \lambda^{2}+3 \lambda-4\right)}{1800 \lambda^{2}-1800 \lambda+480} t^{5}-\frac{\left(-5 \lambda^{3}-15 \lambda^{2}+6 \lambda+2\right)}{1800 \lambda^{2}-1800 \lambda+480} t^{4}- \\
& \frac{\left(10 \lambda^{3}+3 \lambda^{2}-5 \lambda\right)}{1800 \lambda^{2}-1800 \lambda+480} t^{3}-\frac{\left(3 \lambda^{2}-5 \lambda^{3}\right)}{1800 \lambda^{2}-1800 \lambda+480} t^{2}
\end{aligned}
$$


For later references we note that:

$$
\begin{array}{ll}
A_{0}^{\prime \prime}(0)=-\frac{6\left(15 \lambda^{4}+5 \lambda^{3}-6 \lambda+2\right)}{\lambda^{2}\left(15 \lambda^{2}-15 \lambda+4\right)}, & A_{0}^{\prime \prime}(1)=\frac{6\left(15 \lambda^{3}-5 \lambda^{2}-5 \lambda+1\right)(\lambda-1)^{2}}{\lambda^{3}\left(15 \lambda^{2}-15 \lambda+4\right)}, \\
A_{1}^{\prime \prime}(0)=\frac{6\left(15 \lambda^{3}-24 \lambda^{2}+12 \lambda-2\right)}{\lambda^{2}\left(15 \lambda^{2}-15 \lambda+4\right)(\lambda-1)^{3}}, & A_{1}^{\prime \prime}(1)=\frac{6\left(15 \lambda^{3}-21 \lambda^{2}+9 \lambda-1\right)}{\lambda^{3}\left(15 \lambda^{2}-15 \lambda+4\right)(\lambda-1)^{2}}, \\
A_{2}^{\prime \prime}(0)=\frac{6 \lambda^{2}\left(15 \lambda^{3}-40 \lambda^{2}+30 \lambda-6\right)}{\left(15 \lambda^{2}-15 \lambda+4\right)(\lambda-1)^{3}}, & A_{2}^{\prime \prime}(1)=-\frac{6\left(15 \lambda^{4}-65 \lambda^{3}+105 \lambda^{2}-69 \lambda+16\right)}{\left(15 \lambda^{2}-15 \lambda+4\right)(\lambda-1)^{2}}, \\
A_{3}^{\prime \prime}(0)=-\frac{12\left(5 \lambda^{3}-3 \lambda+1\right)}{\lambda\left(15 \lambda^{2}-15 \lambda+4\right)}, & A_{3}^{\prime \prime}(1)=\frac{6\left(5 \lambda^{2}-5 \lambda+1\right)(\lambda-1)^{2}}{\lambda^{2}\left(15 \lambda^{2}-15 \lambda+4\right)}, \\
A_{4}^{\prime \prime}(0)=-\frac{6 \lambda^{2}\left(5 \lambda^{2}-5 \lambda+1\right)}{\left(15 \lambda^{2}-15 \lambda+4\right)(\lambda-1)^{2}}, & A_{4}^{\prime \prime}(1)=\frac{12\left(5 \lambda^{3}-15 \lambda^{2}+12 \lambda-3\right)}{(\lambda-1)\left(15 \lambda^{2}-15 \lambda+4\right)}, \\
A_{5}^{\prime \prime}(0)=-\frac{5 \lambda-2}{(\lambda-1)\left(15 \lambda^{2}-15 \lambda+4\right)}, & A_{5}^{\prime \prime}(1)=-\frac{5 \lambda-3}{\lambda\left(15 \lambda^{2}-15 \lambda+4\right)}, \\
A_{6}^{\prime \prime}(0)=\frac{\lambda^{2}(5 \lambda-3)}{60\left(15 \lambda^{2}-15 \lambda+4\right),} & A_{6}^{\prime \prime}(1)=\frac{(5 \lambda-2)(\lambda-1)^{2}}{60\left(15 \lambda^{2}-15 \lambda+4\right)}, \\
A_{0}^{(6)}(0)=A_{0}^{(6)}(1)=-\frac{720\left(6 \lambda^{2}+3 \lambda-1\right)}{\lambda\left(15 \lambda^{4}-15 \lambda^{3}+4 \lambda^{2}\right)}, & \\
A_{1}^{(6)}(0)=A_{1}^{(6)}(1)=\frac{120\left(6 \lambda^{2}-6 \lambda+1\right)}{\lambda\left(-15 \lambda^{7}+60 \lambda^{6}-94 \lambda^{5}+72 \lambda^{4}-27 \lambda^{3}+4 \lambda^{2}\right)}, \\
A_{2}^{(6)}(0)=A_{2}^{(6)}(1)=\frac{\left(6 \lambda^{2}-15 \lambda+8\right)}{15 \lambda^{5}-60 \lambda^{4}+94 \lambda^{3}-72 \lambda^{2}+27 \lambda-4}, & \\
A_{3}^{(6)}(0)=A_{3}^{(6)}(1)=-\frac{(3 \lambda-1)}{\lambda\left(15 \lambda^{3}-15 \lambda^{2}+4 \lambda\right)}, & \\
A_{6}^{(6)}(0)=A_{6}^{(6)}(1)=\frac{(2 \lambda-1)}{900 \lambda^{2}-900 \lambda+240}, & \\
A_{4}^{(6)}(0)=A_{4}^{(6)}(1)=-\frac{(3 \lambda-2)}{15 \lambda^{4}-45 \lambda^{3}+49 \lambda^{2}-23 \lambda+4}, & \\
A_{5}^{(6)}(0)=A_{5}^{(6)}(1)=-\frac{1}{2 \lambda\left(15 \lambda^{3}-30 \lambda^{2}+19 \lambda-4\right)}, & \\
& \\
& \\
&
\end{array}
$$

For $f \in C^{6}[0,1]$,we have the following expansions on $\left[x_{i}, x_{i+1}\right]$

$$
\begin{aligned}
f(x)= & f\left(x_{i}\right)+\left(x-x_{i}\right) f^{\prime}\left(x_{i}\right)+\frac{\left(x-x_{i}\right)^{2}}{2 !} f^{\prime \prime}\left(x_{i}\right)+\frac{\left(x-x_{i}\right)^{3}}{3 !} f^{\prime \prime \prime}\left(x_{i}\right)+\frac{\left(x-x_{i}\right)^{4}}{4 !} f^{(4)}\left(x_{i}\right)+ \\
& \frac{\left(x-x_{i}\right)^{5}}{5 !} f^{(5)}\left(x_{i}\right)+\frac{\left(x-x_{i}\right)^{6}}{6 !} f^{(6)}(\theta), \text { for } x<\theta<x_{i},
\end{aligned}
$$




$$
\begin{aligned}
f\left(x_{i-1}\right)= & f\left(x_{i}\right)+\left(x_{i-1}-x_{i}\right) f^{\prime}\left(x_{i}\right)+\frac{\left(x_{i-1}-x_{i}\right)^{2}}{2 !} f^{\prime \prime}\left(x_{i}\right)+\frac{\left(x_{i-1}-x_{i}\right)^{3}}{3 !} f^{\prime \prime \prime}\left(x_{i}\right)+ \\
& +\frac{\left(x_{i-1}-x_{i}\right)^{4}}{4 !} f^{(4)}\left(x_{i}\right) \frac{\left(x_{i-1}-x_{i}\right)^{5}}{5 !} f^{(5)}\left(x_{i}\right)+\frac{\left(x-x_{i}\right)^{6}}{6 !} f^{(6)}\left(\theta_{1, i}\right), \text { for } x_{i-1}<\theta_{1, i}<x_{i}, \\
f\left(x_{i-1}\right)= & f\left(x_{i}\right)-h f^{\prime}\left(x_{i}\right)+\frac{h^{2}}{2 !} f^{\prime \prime}\left(x_{i}\right)-\frac{h^{3}}{3 !} f^{\prime \prime \prime}\left(x_{i}\right)+\frac{h^{4}}{4 !} f^{(4)}\left(x_{i}\right)-\frac{h^{5}}{5 !} f^{(5)}\left(x_{i}\right)+\frac{h^{6}}{6 !} f^{(6)}\left(\theta_{1, i}\right),
\end{aligned}
$$

for $x_{i-1}<\theta_{1, i}<x_{i}$,

$$
f\left(x_{i+1}\right)=f\left(x_{i}\right)+h f^{\prime}\left(x_{i}\right)+\frac{h^{2}}{2 !} f^{\prime \prime}\left(x_{i}\right)+\frac{h^{3}}{3 !} f^{\prime \prime \prime}\left(x_{i}\right)+\frac{h^{4}}{4 !} f^{(4)}\left(x_{i}\right)+\frac{h^{5}}{5 !} f^{(5)}\left(x_{i}\right)+\frac{h^{6}}{6 !} f^{(6)}\left(\theta_{2, i}\right),
$$$$
\text { for } x_{i}<\theta_{2, i}<x_{i+1} \text {, }
$$

$$
\begin{aligned}
f\left(x_{i-1+\lambda}\right) & =f\left(x_{i}\right)+(\lambda-1) h f^{\prime}\left(x_{i}\right)+\frac{(\lambda-1)^{2} h^{2}}{2 !} f^{\prime \prime}\left(x_{i}\right)+\frac{(\lambda-1)^{3} h^{3}}{3 !} f^{\prime \prime \prime}\left(x_{i}\right)+ \\
& \frac{(\lambda-1)^{4} h^{4}}{4 !} f^{(4)}\left(x_{i}\right)+\frac{(\lambda-1)^{5} h^{5}}{5 !} f^{(5)}\left(x_{i}\right)+\frac{(\lambda-1)^{6} h^{6}}{6 !} f^{(6)}\left(\theta_{3, i}\right), \text { for } x_{i}<\theta_{3, i}<x_{i-1+\lambda,},
\end{aligned}
$$$$
f\left(x_{i+\lambda}\right)=f\left(x_{i}\right)+\lambda h f^{\prime}\left(x_{i}\right)+\frac{\lambda^{2} h^{2}}{2 !} f^{\prime \prime}\left(x_{i}\right)+\frac{\lambda^{3} h^{3}}{3 !} f^{\prime \prime \prime}\left(x_{i}\right)+\frac{\lambda^{4} h^{4}}{4 !} f^{(4)}\left(x_{i}\right)+\frac{\lambda^{5} h^{5}}{5 !} f^{(5)}\left(x_{i}\right)+
$$
$\frac{\lambda^{6} h^{6}}{6 !} f^{(6)}\left(\theta_{4, i}\right)$, for $x_{i}<\theta_{4, i}<x_{i+\lambda}$,

$$
f^{\prime \prime}\left(x_{i-1+\lambda}\right)=f^{\prime \prime}\left(x_{i}\right)+(\lambda-1) h f^{\prime \prime \prime}\left(x_{i}\right)+\frac{(\lambda-1)^{2} h^{2}}{2 !} f^{(4)}\left(x_{i}\right)+\frac{(\lambda-1)^{3} h^{3}}{3 !} f^{(5)}\left(x_{i}\right)+
$$

$$
\frac{(\lambda-1)^{4} h^{4}}{4 !} f^{(6)}\left(\theta_{5, i}\right) \text {, for } x_{i}<\theta_{5, i}<x_{i-1+\lambda}
$$

$$
f^{\prime \prime}\left(x_{i+\lambda}\right)=f^{\prime \prime}\left(x_{i}\right)+\lambda h f^{\prime \prime \prime}\left(x_{i}\right)+\frac{\lambda^{2} h^{2}}{2 !} f^{(4)}\left(x_{i}\right)+\frac{\lambda^{3} h^{3}}{3 !} f^{(5)}\left(x_{i}\right)+\frac{\lambda^{4} h^{4}}{4 !} f^{(6)}\left(\theta_{6, i}\right),
$$

for $x_{i}<\theta_{6, i}<x_{i+\lambda}$.

$$
\begin{aligned}
& f^{(5)}\left(x_{i-1+\lambda}\right)=f^{(5)}\left(x_{i}\right)+(\lambda-1) h f^{(6)}\left(\theta_{7, i}\right), \text { for } x_{i}<\theta_{7, i}<x_{i-1+\lambda}, \\
& f^{(5)}\left(x_{i+\lambda}\right)=f^{(5)}\left(x_{i}\right)+\lambda h f^{(6)}\left(\theta_{8, i}\right), \text { for } x_{i}<\theta_{8, i}<x_{i+\lambda}, \\
& f^{\prime}\left(x_{i-1}\right)=f^{\prime}\left(x_{i}\right)-h f^{\prime \prime}\left(x_{i}\right)+\frac{h^{2}}{2 !} f^{\prime \prime \prime}\left(x_{i}\right)-\frac{h^{3}}{3 !} f^{(4)}\left(x_{i}\right)+\frac{h^{4}}{4 !} f^{(5)}\left(x_{i}\right)-\frac{h^{5}}{5 !} f^{(6)}\left(\theta_{9, i}\right),
\end{aligned}
$$

for $x_{i-1}<\theta_{9, i}<x_{i}$

$f^{\prime}\left(x_{i+1}\right)=f^{\prime}\left(x_{i}\right)+h f^{\prime \prime}\left(x_{i}\right)+\frac{h^{2}}{2 !} f^{\prime \prime \prime}\left(x_{i}\right)+\frac{h^{3}}{3 !} f^{(4)}\left(x_{i}\right)+\frac{h^{4}}{4 !} f^{(5)}\left(x_{i}\right)+\frac{h^{5}}{5 !} f^{(6)}\left(\theta_{10, i}\right)$,

$$
\text { for } x_{i}<\theta_{10, i}<x_{i+1}
$$




\subsection{Existences and Uniqueness Theorems:}

In this section, the existence and uniqueness theorem for spline function of degree six which interpolate the Lacunary data $(0,2,5)$ is presented and examined.

\section{Theorem 1: New Existence and Uniqueness of the Spline Function}

For given arbitrary numbers $f\left(x_{i}\right), f^{(r)}\left(x_{i+\lambda}\right), i=0,1, \ldots, n-1 ; r=0,2,5$ and $f^{\prime \prime}\left(x_{0}\right), f^{\prime \prime}\left(x_{n}\right)$,There exists a unique spline $S_{n}(x) \in S_{n, 6}^{2}$

such that :

$$
\begin{aligned}
& S_{n}\left(x_{i}\right)=f\left(x_{i}\right), i=0,1, \ldots, n-1, \\
& S_{n}{ }^{(r)}\left(x_{i+\lambda}\right)=f^{(r)}\left(x_{i+\lambda}\right), i=0,1, \ldots, n-1 ; r=0,2,5, \\
& S_{n}^{\prime \prime}\left(x_{0}\right)=f^{\prime \prime}\left(x_{0}\right), S_{n}^{\prime \prime}\left(x_{n}\right)=f^{\prime \prime}\left(x_{n}\right) .
\end{aligned}
$$

\section{Theorem 2:}

Let $f(x) \in C^{6}[0,1]$ and $S_{n}(x) \in S_{n, 6}^{2}$ be a unique spline satisfying the conditions of Theorem 1, then

$$
\left\|S_{n}^{(r)}(x)-f^{(r)}(x)\right\| \leq K m^{r-6} w\left(f^{(6)} ; \frac{1}{m}\right) ; r=0,1,2,3,4,5
$$

Where $m=\frac{1}{h}$

$$
\begin{aligned}
& K=\frac{1}{10800 \lambda^{2}\left(15 \lambda^{2}-15 \lambda+4\right)\left(30 \lambda^{6}-90 \lambda^{5}+110 \lambda^{4}-70 \lambda^{3}+27 \lambda^{2}-7 \lambda+1\right)(\lambda-1)^{2}} \\
& \left(20250 \lambda^{14}-114150 \lambda^{13}+339000 \lambda^{12}-635430 \lambda^{11}+778215 \lambda^{10}-604405 \lambda^{9}+245160 \lambda^{8}\right. \\
& +34920 \lambda^{7}-126180 \lambda^{6}+96086 \lambda^{5}-42587 \lambda^{4}+11021 \lambda^{3}-1129 \lambda^{2}-135 \lambda \\
& +33)
\end{aligned}
$$




\section{Proof of the theorem 1:}

The proof depends on the following representations of $S_{n}(x)$,

for $x_{i} \leq x \leq x_{i+1} i=0,1, \ldots, m-1$, we have

$$
\begin{aligned}
S_{n}(x)=f\left(x_{i}\right) & A_{0}\left(\frac{x-x_{i}}{h}\right)+f\left(x_{i+\lambda}\right) A_{1}\left(\frac{x-x_{i}}{h}\right)+f\left(x_{i+1}\right) A_{2}\left(t \frac{x-x_{i}}{h}\right)+h S_{i}^{\prime}\left(x_{i}\right) A_{3}\left(\frac{x-x_{i}}{h}\right) \\
+ & h S_{i}^{\prime}\left(x_{i+1}\right) A_{4}\left(\frac{x-x_{i}}{h}\right)+h^{2} f^{\prime \prime}\left(x_{i+\lambda}\right) A_{5}\left(\frac{x-x_{i}}{h}\right)+h^{5} f^{(5)}\left(x_{i+\lambda}\right) A_{6}\left(\frac{x-x_{i}}{h}\right)
\end{aligned}
$$

On using equation (8) and the conditions

$$
S_{n}^{\prime \prime}(0)=f^{\prime \prime}(0), S_{n}^{\prime \prime}(1)=f^{\prime \prime}(1)
$$

We see that $\mathrm{S}_{\mathrm{n}}(\mathrm{x})$ as given by (8) satisfies (2) and is sixtic in $\left[x_{i}, x_{i+1}\right], \mathrm{i}=0,1, \ldots, \mathrm{m}-1$. We also need to show that whether it is possible to determine $S_{n}^{\prime}\left(x_{i}\right), i=1,2, \ldots, m-1$ uniquely. For this purpose we use the fact that $S_{n}(x) \in C^{2}[0,1]$ and therefore the conditions:

$S_{n}^{\prime \prime}\left(x_{i+}\right)=S_{n}^{\prime \prime}\left(x_{i-}\right), i=1,2, \ldots, m-1$

Where $S_{n}^{\prime \prime}\left(x_{i+}\right)=\lim _{x \rightarrow x_{i}^{+}} S_{n}^{\prime \prime}(x)$, and $S_{n}^{\prime \prime}\left(x_{i-}\right)=\lim _{x \rightarrow x_{i}^{-}} S_{n}^{\prime \prime}(x)$,

with the help of (8) and (9) reduced to

$$
\begin{aligned}
& 6 \lambda\left(5 \lambda^{2}-5 \lambda+1\right)(\lambda-1)^{5} h S_{i}^{\prime}\left(x_{i-1}\right)+\lambda^{2}(\lambda-1)^{2}\left(120 \lambda^{4}-240 \lambda^{3}+108 \lambda^{2}+12 \lambda-12\right) \\
& h S_{i}^{\prime}\left(x_{i}\right)+6 \lambda^{5}(\lambda-1)\left(5 \lambda^{2}-5 \lambda+1\right) h S_{i}^{\prime}\left(x_{i+1}\right)=-6\left(15 \lambda^{3}-5 \lambda^{2}-5 \lambda+1\right)(\lambda-1)^{5} f\left(x_{i-1}\right) \\
& +12 \lambda(\lambda-1)\left(-20 \lambda^{5}+50 \lambda^{4}-34 \lambda^{3}+\lambda^{2}+5 \lambda-1\right) f\left(x_{i}\right)+6 \lambda^{5}\left(15 \lambda^{3}-40 \lambda^{2}+30 \lambda-6\right) \\
& f\left(x_{i+1}\right)-6(\lambda-1)\left(15 \lambda^{3}-21 \lambda^{2}+9 \lambda-1\right) f\left(x_{i-1+\lambda}\right)+6 \lambda\left(15 \lambda^{3}-24 \lambda^{2}+12 \lambda-2\right) f\left(x_{i+\lambda}\right) \\
& +\lambda^{2}(\lambda-1)^{3}(5 \lambda-3) h^{2} f^{\prime \prime}\left(x_{i-1+\lambda}\right)-\lambda^{3}(\lambda-1)^{2}(5 \lambda-2) h^{2} f^{\prime \prime}\left(x_{i+\lambda}\right) \\
& -\frac{1}{60}(5 \lambda-2) \lambda^{3}(\lambda-1)^{5} h^{5} f^{(5)}\left(x_{i-1+\lambda}\right)+\frac{1}{60} \lambda^{5}(\lambda-1)^{3}(5 \lambda-3) h^{5} f^{(5)}\left(x_{i+\lambda}\right) \\
& \quad \text { for } i=1,2, \ldots, m-1
\end{aligned}
$$


Equation (11) is a strictly tri-diagonal dominant system which has a unique solution. Thus $S_{n}^{\prime \prime}\left(x_{i}\right), \mathrm{i}=1,2, \ldots, \mathrm{m}-1$ can be obtained uniquely by the system (11) which established Theorem 1.

\subsection{Convergence and Error Bounds:}

In this section, the upper bounds for errors studied first help the results of the following:

\section{Lemma 1:}

Let us write $B_{i}=\left|S_{n}^{\prime}\left(x_{i}\right)-f^{\prime}\left(x_{i}\right)\right|$, then for $f \in C^{6}[0,1]$, we have

$$
\max B_{i} \leq \frac{2(2 \lambda-1)\left(2 \lambda^{2}-2 \lambda+1\right)\left(5 \lambda^{2}-5 \lambda+1\right)}{\left(30 \lambda^{6}-90 \lambda^{5}+110 \lambda^{4}-70 \lambda^{3}+27 \lambda^{2}-7 \lambda+1\right)} \frac{h^{5}}{5 !} W\left(f^{(6)} ; \frac{1}{m}\right) \quad i=1,2, \ldots, m-1
$$

Where $\lambda \in(0,1)$

\section{Proof:}

From (6) and (11)

$$
\begin{aligned}
& \left(S_{i}^{\prime}\left(x_{i-1}\right)-f^{\prime}\left(x_{i-1}\right)\right) A+\left(S_{i}^{\prime}\left(x_{i}\right)-f^{\prime}\left(x_{i}\right)\right) B+\left(S_{i}^{\prime}\left(x_{i+1}\right)-f^{\prime}\left(x_{i+1}\right)\right) C=\left(S_{i}^{\prime}\left(x_{i-1}\right) A+S_{i}^{\prime}\left(x_{i}\right) B+\right. \\
& \left.S_{i}^{\prime}\left(x_{i+1}\right) C\right)-\left(f^{\prime}\left(x_{i-1}\right) A+f^{\prime}\left(x_{i}\right) B+f^{\prime}\left(x_{i+1}\right) C\right)=-\frac{1}{h}\left[f ( x _ { i - 1 } ) \left(90 \lambda^{8}-480 \lambda^{7}+1020 \lambda^{6}-\right.\right. \\
& \left.1044 \lambda^{5}+420 \lambda^{4}+120 \lambda^{3}-180 \lambda^{2}+60 \lambda-6\right)-f\left(x_{i}\right)\left(-240 \lambda^{7}+840 \lambda^{6}-1008 \lambda^{5}+\right. \\
& \left.420 \lambda^{4}+48 \lambda^{3}-72 \lambda^{2}+12 \lambda\right)-f\left(x_{i+1}\right)\left(90 \lambda^{8}-240 \lambda^{7}+180 \lambda^{6}-36 \lambda^{5}\right)+ \\
& f\left(x_{i-1+\lambda}\right)\left(90 \lambda^{4}-216 \lambda^{3}+180 \lambda^{2}-60 \lambda+6\right)-f\left(x_{i+\lambda}\right)\left(90 \lambda^{4}-144 \lambda^{3}+72 \lambda^{2}-12 \lambda\right)- \\
& h^{2} f^{\prime \prime}\left(x_{i-1+\lambda}\right)\left(5 \lambda^{6}-18 \lambda^{5}+24 \lambda^{4}-14 \lambda^{3}+3 \lambda^{2}\right)+h^{2} f^{\prime \prime}\left(x_{i+\lambda}\right)\left(5 \lambda^{6}-12 \lambda^{5}+9 \lambda^{4}-2 \lambda^{3}\right)+ \\
& \left.h^{5} f^{(5)}\left(x_{i-1+\lambda}\right)\left(\frac{\lambda^{9}}{12}-\frac{9 \lambda^{8}}{20}+\lambda^{7}-\frac{7 \lambda^{6}}{6}+\frac{3 \lambda^{5}}{4}-\frac{\lambda^{4}}{4}+\frac{\lambda^{3}}{30}\right)-h^{5} f^{(5)}\left(x_{i+\lambda}\right)\left(\frac{\lambda^{9}}{12}-\frac{3 \lambda^{8}}{10}+\frac{2 \lambda^{7}}{5}-\frac{7 \lambda^{6}}{30}+\frac{\lambda^{5}}{20}\right)\right]- \\
& -\left(f^{\prime}\left(x_{i-1}\right) A+f^{\prime}\left(x_{i}\right) B+f^{\prime}\left(x_{i+1}\right) C\right) \\
& =\left(-\frac{1}{h}\right)\left(\left(90 \lambda^{8}-480 \lambda^{7}+1020 \lambda^{6}-1044 \lambda^{5}+420 \lambda^{4}+120 \lambda^{3}-180 \lambda^{2}+60 \lambda-6\right)-\right. \\
& \left(-240 \lambda^{7}+840 \lambda^{6}-1008 \lambda^{5}+420 \lambda^{4}+48 \lambda^{3}-72 \lambda^{2}+12 \lambda\right)-\left(90 \lambda^{8}-240 \lambda^{7}+180 \lambda^{6}-\right. \\
& \left.\left.36 \lambda^{5}\right)+\left(90 \lambda^{4}-216 \lambda^{3}+180 \lambda^{2}-60 \lambda+6\right)-\left(90 \lambda^{4}-144 \lambda^{3}+72 \lambda^{2}-12 \lambda\right)\right) f\left(x_{i}\right) \\
& +\left(( - \frac { 1 } { h } ) \left(-h\left(90 \lambda^{8}-480 \lambda^{7}+1020 \lambda^{6}-1044 \lambda^{5}+420 \lambda^{4}+120 \lambda^{3}-180 \lambda^{2}+60 \lambda-6\right)-\right.\right. \\
& h\left(90 \lambda^{8}-240 \lambda^{7}+180 \lambda^{6}-36 \lambda^{5}\right)+(\lambda-1) h\left(90 \lambda^{4}-216 \lambda^{3}+180 \lambda^{2}-60 \lambda+6\right)- \\
& \left.\lambda h\left(90 \lambda^{4}-144 \lambda^{3}+72 \lambda^{2}-12 \lambda\right)\right)-\left(30 \lambda^{8}-180 \lambda^{7}+456 \lambda^{6}-630 \lambda^{5}+510 \lambda^{4}-240 \lambda^{3}+\right. \\
& \left.60 \lambda^{2}-6 \lambda\right)-\left(120 \lambda^{8}-480 \lambda^{7}+708 \lambda^{6}-444 \lambda^{5}+72 \lambda^{4}+36 \lambda^{3}-12 \lambda^{2}\right)-\left(30 \lambda^{8}-\right. \\
& \left.\left.60 \lambda^{7}+36 \lambda^{6}-6 \lambda^{5}\right)\right) f^{\prime}\left(x_{i}\right)
\end{aligned}
$$




$$
\begin{aligned}
& +\left(( - \frac { 1 } { h } ) \left(\frac{h^{2}}{2 !}\left(90 \lambda^{8}-480 \lambda^{7}+1020 \lambda^{6}-1044 \lambda^{5}+420 \lambda^{4}+120 \lambda^{3}-180 \lambda^{2}+60 \lambda-6\right)-\right.\right. \\
& \frac{h^{2}}{2 !}\left(90 \lambda^{8}-240 \lambda^{7}+180 \lambda^{6}-36 \lambda^{5}\right)+\frac{(\lambda-1)^{2} h^{2}}{2 !}\left(90 \lambda^{4}-216 \lambda^{3}+180 \lambda^{2}-60 \lambda+6\right)- \\
& \frac{\lambda^{2} h^{2}}{2 !}\left(90 \lambda^{4}-144 \lambda^{3}+72 \lambda^{2}-12 \lambda\right)-h^{2}\left(5 \lambda^{6}-18 \lambda^{5}+24 \lambda^{4}-14 \lambda^{3}+3 \lambda^{2}\right)+h^{2}\left(5 \lambda^{6}-\right. \\
& \left.\left.12 \lambda^{5}+9 \lambda^{4}-2 \lambda^{3}\right)\right)+h\left(30 \lambda^{8}-180 \lambda^{7}+456 \lambda^{6}-630 \lambda^{5}+510 \lambda^{4}-240 \lambda^{3}+60 \lambda^{2}-\right. \\
& \left.6 \lambda)-h\left(30 \lambda^{8}-60 \lambda^{7}+36 \lambda^{6}-6 \lambda^{5}\right)\right) f^{\prime \prime}\left(x_{i}\right) \\
& +\left(( - \frac { 1 } { h } ) \left(-\frac{h^{3}}{3 !}\left(90 \lambda^{8}-480 \lambda^{7}+1020 \lambda^{6}-1044 \lambda^{5}+420 \lambda^{4}+120 \lambda^{3}-180 \lambda^{2}+60 \lambda-6\right)-\right.\right. \\
& \frac{h^{3}}{3 !}\left(90 \lambda^{8}-240 \lambda^{7}+180 \lambda^{6}-36 \lambda^{5}\right)+\frac{(\lambda-1)^{3} h^{3}}{3 !}\left(90 \lambda^{4}-216 \lambda^{3}+180 \lambda^{2}-60 \lambda+6\right)- \\
& \frac{\lambda^{3} h^{3}}{3 !}\left(90 \lambda^{4}-144 \lambda^{3}+72 \lambda^{2}-12 \lambda\right)-h^{2}(\lambda-1) h\left(5 \lambda^{6}-18 \lambda^{5}+24 \lambda^{4}-14 \lambda^{3}+3 \lambda^{2}\right)+ \\
& \left.h^{2} \lambda h\left(5 \lambda^{6}-12 \lambda^{5}+9 \lambda^{4}-2 \lambda^{3}\right)\right)-\frac{h^{2}}{2 !}\left(30 \lambda^{8}-180 \lambda^{7}+456 \lambda^{6}-630 \lambda^{5}+510 \lambda^{4}-240 \lambda^{3}+\right. \\
& \left.\left.60 \lambda^{2}-6 \lambda\right)-\frac{h^{2}}{2 !}\left(30 \lambda^{8}-60 \lambda^{7}+36 \lambda^{6}-6 \lambda^{5}\right)\right) f^{\prime \prime \prime}\left(x_{i}\right) \\
& +\left(( - \frac { 1 } { h } ) \left(\frac{h^{4}}{4 !}\left(90 \lambda^{8}-480 \lambda^{7}+1020 \lambda^{6}-1044 \lambda^{5}+420 \lambda^{4}+120 \lambda^{3}-180 \lambda^{2}+60 \lambda-6\right)-\right.\right. \\
& \frac{h^{4}}{4 !}\left(90 \lambda^{8}-240 \lambda^{7}+180 \lambda^{6}-36 \lambda^{5}\right)+\frac{(\lambda-1)^{4} h^{4}}{4 !}\left(90 \lambda^{4}-216 \lambda^{3}+180 \lambda^{2}-60 \lambda+6\right)- \\
& \frac{\lambda^{4} h^{4}}{4 !}\left(90 \lambda^{4}-144 \lambda^{3}+72 \lambda^{2}-12 \lambda\right)-h^{2} \frac{(\lambda-1)^{2} h^{2}}{2 !}\left(5 \lambda^{6}-18 \lambda^{5}+24 \lambda^{4}-14 \lambda^{3}+3 \lambda^{2}\right)+ \\
& \left.h^{2} \frac{\lambda^{2} h^{2}}{2 !}\left(5 \lambda^{6}-12 \lambda^{5}+9 \lambda^{4}-2 \lambda^{3}\right)\right)+\frac{h^{3}}{3 !}\left(30 \lambda^{8}-180 \lambda^{7}+456 \lambda^{6}-630 \lambda^{5}+510 \lambda^{4}-\right. \\
& \left.\left.240 \lambda^{3}+60 \lambda^{2}-6 \lambda\right)-\frac{h^{3}}{3 !}\left(30 \lambda^{8}-60 \lambda^{7}+36 \lambda^{6}-6 \lambda^{5}\right)\right) f^{(4)}\left(x_{i}\right) \\
& +\left(( - \frac { 1 } { h } ) \left(-\frac{h^{5}}{5 !}\left(90 \lambda^{8}-480 \lambda^{7}+1020 \lambda^{6}-1044 \lambda^{5}+420 \lambda^{4}+120 \lambda^{3}-180 \lambda^{2}+60 \lambda-6\right)-\right.\right. \\
& \frac{h^{5}}{5 !}\left(90 \lambda^{8}-240 \lambda^{7}+180 \lambda^{6}-36 \lambda^{5}\right)+\frac{(\lambda-1)^{5} h^{5}}{5 !}\left(90 \lambda^{4}-216 \lambda^{3}+180 \lambda^{2}-60 \lambda+6\right)- \\
& \frac{\lambda^{5} h^{5}}{5 !}\left(90 \lambda^{4}-144 \lambda^{3}+72 \lambda^{2}-12 \lambda\right)-h^{2} \frac{(\lambda-1)^{3} h^{3}}{3 !}\left(5 \lambda^{6}-18 \lambda^{5}+24 \lambda^{4}-14 \lambda^{3}+3 \lambda^{2}\right)+ \\
& h^{2} \frac{\lambda^{3} h^{3}}{3 !}\left(5 \lambda^{6}-12 \lambda^{5}+9 \lambda^{4}-2 \lambda^{3}\right)+h^{5}\left(\frac{\lambda^{9}}{12}-\frac{9 \lambda^{8}}{20}+\lambda^{7}-\frac{7 \lambda^{6}}{6}+\frac{3 \lambda^{5}}{4}-\frac{\lambda^{4}}{4}+\frac{\lambda^{3}}{30}\right)-h^{5}\left(\frac{\lambda^{9}}{12}-\frac{3 \lambda^{8}}{10}+\right. \\
& \left.\left.\frac{2 \lambda^{7}}{5}-\frac{7 \lambda^{6}}{30}+\frac{\lambda^{5}}{20}\right)\right)-\frac{h^{4}}{4 !}\left(30 \lambda^{8}-180 \lambda^{7}+456 \lambda^{6}-630 \lambda^{5}+510 \lambda^{4}-240 \lambda^{3}+60 \lambda^{2}-6 \lambda\right)- \\
& \left.\frac{h^{4}}{4 !}\left(30 \lambda^{8}-60 \lambda^{7}+36 \lambda^{6}-6 \lambda^{5}\right)\right) f^{(5)}\left(x_{i}\right)
\end{aligned}
$$


$+\frac{h^{5}}{5 !}\left(\frac{1}{6}\left(90 \lambda^{8}-480 \lambda^{7}+1020 \lambda^{6}-1044 \lambda^{5}+420 \lambda^{4}+120 \lambda^{3}-180 \lambda^{2}+60 \lambda-\right.\right.$

6) $f^{(6)}\left(\theta_{1, i}\right)-\frac{1}{6}\left(90 \lambda^{8}-240 \lambda^{7}+180 \lambda^{6}-36 \lambda^{5}\right) f^{(6)}\left(\theta_{2, i}\right)+\frac{(\lambda-1)^{6}}{6}\left(90 \lambda^{4}-216 \lambda^{3}+180 \lambda^{2}-\right.$ $60 \lambda+6) f^{(6)}\left(\theta_{3, i}\right)-\frac{\lambda^{6}}{6}\left(90 \lambda^{4}-144 \lambda^{3}+72 \lambda^{2}-12 \lambda\right) f^{(6)}\left(\theta_{4, i}\right)-5(\lambda-1)^{4}\left(5 \lambda^{6}-18 \lambda^{5}+\right.$ $\left.24 \lambda^{4}-14 \lambda^{3}+3 \lambda^{2}\right) f^{(6)}\left(\theta_{5, i}\right)+5 \lambda^{4}\left(5 \lambda^{6}-12 \lambda^{5}+9 \lambda^{4}-2 \lambda^{3}\right) f^{(6)}\left(\theta_{6, i}\right)+5 !(\lambda-1)\left(\frac{\lambda^{9}}{12}-\right.$

$\left.\frac{9 \lambda^{8}}{20}+\lambda^{7}-\frac{7 \lambda^{6}}{6}+\frac{3 \lambda^{5}}{4}-\frac{\lambda^{4}}{4}+\frac{\lambda^{3}}{30}\right) f^{(6)}\left(\theta_{7, i}\right)-5 ! \lambda\left(\frac{\lambda^{9}}{12}-\frac{3 \lambda^{8}}{10}+\frac{2 \lambda^{7}}{5}-\frac{7 \lambda^{6}}{30}+\frac{\lambda^{5}}{20}\right) f^{(6)}\left(\theta_{6, i}\right)+$ $\left(30 \lambda^{8}-180 \lambda^{7}+456 \lambda^{6}-630 \lambda^{5}+510 \lambda^{4}-240 \lambda^{3}+60 \lambda^{2}-6 \lambda\right) f^{(6)}\left(\theta_{9, i}\right)-\left(30 \lambda^{8}-\right.$ $\left.\left.60 \lambda^{7}+36 \lambda^{6}-6 \lambda^{5}\right) f^{(6)}\left(\theta_{10, i}\right)\right)$

$\leq-12 \lambda(2 \lambda-1)(\lambda-1)\left(2 \lambda^{2}-2 \lambda+1\right)\left(5 \lambda^{2}-5 \lambda+1\right) \frac{h^{5}}{5 !} W\left(f^{(6)} ; \frac{1}{m}\right)$.

So we have

$\max \left|S^{\prime}(x)-f^{\prime}(x)\right| \leq \frac{2(2 \lambda-1)\left(2 \lambda^{2}-2 \lambda+1\right)\left(5 \lambda^{2}-5 \lambda+1\right)}{\left(30 \lambda^{6}-90 \lambda^{5}+110 \lambda^{4}-70 \lambda^{3}+27 \lambda^{2}-7 \lambda+1\right)} \frac{h^{5}}{5 !} \alpha_{1} W\left(f^{(6)} ; \frac{1}{m}\right)$.

Where $\left|\alpha_{1}\right| \leq 1, i=1,2, \ldots, m-1$

The result (12) follows on using the property of diagonal dominant.

\section{Lemma 2:}

Let $f \in C^{6}[0,1]$ then

$$
\begin{aligned}
& \left|S_{n}^{(5)}\left(x_{i}\right)-f^{(5)}\left(x_{i}\right)\right| \leq \frac{12(2 \lambda-1)^{2}\left(2 \lambda^{2}-2 \lambda+1\right)\left(3 \lambda^{2}-3 \lambda+1\right)\left(5 \lambda^{2}-5 \lambda+1\right)}{\lambda(\lambda-1)^{2}\left(15 \lambda^{2}-15 \lambda+4\right)\left(30 \lambda^{6}-90 \lambda^{5}+110 \lambda^{4}-70 \lambda^{3}+27 \lambda^{2}-7 \lambda+1\right)} h W\left(f^{(6)} ; \frac{1}{m}\right) \\
& \left|S_{n}^{(5)}\left(x_{i+1}\right)-f^{(5)}\left(x_{i}\right)\right| \leq \frac{2(2 \lambda-1)^{2}\left(2 \lambda^{2}-2 \lambda+1\right)\left(3 \lambda^{2}-3 \lambda+1\right)\left(5 \lambda^{2}-5 \lambda+1\right)}{\lambda^{2}(\lambda-1)\left(15 \lambda^{2}-15 \lambda+4\right)\left(30 \lambda^{6}-90 \lambda^{5}+110 \lambda^{4}-70 \lambda^{3}+27 \lambda^{2}-7 \lambda+1\right)} h W\left(f^{(6)} ; \frac{1}{m}\right)
\end{aligned}
$$

$$
\begin{aligned}
&\left|S_{n}^{(5)}\left(x_{i+\lambda}\right)-f^{(5)}\left(x_{i}\right)\right| \leq \lambda h W\left(f^{(6)} ; \frac{1}{m}\right) \\
&\left|S_{\lambda}^{(4)}\left(x_{i+\lambda}\right)-f^{(4)}\left(x_{i+\lambda}\right)\right| \leq \\
& \frac{(4 \lambda-2)\left(2 \lambda^{2}-2 \lambda+1\right)\left(5 \lambda^{2}-5 \lambda+1\right)\left(10 \lambda^{5}-25 \lambda^{4}+30 \lambda^{3}-20 \lambda^{2}+7 \lambda-1\right)}{5 \lambda^{2}(\lambda-1)^{2}\left(15 \lambda^{2}-15 \lambda+4\right)\left(30 \lambda^{6}-90 \lambda^{5}+110 \lambda^{4}-70 \lambda^{3}+27 \lambda^{2}-7 \lambda+1\right)} \frac{h^{2}}{2 !} W\left(f^{(6)} ; \frac{1}{m}\right)
\end{aligned}
$$


$\left|S_{\lambda}^{(3)}\left(x_{i+\lambda}\right)-f^{(3)}\left(x_{i+\lambda}\right)\right| \leq$

$$
\left[3 \lambda^{2}+\frac{(4 \lambda-2)\left(2 \lambda^{2}-2 \lambda+1\right)\left(5 \lambda^{2}-5 \lambda+1\right)\left(15 \lambda^{6}-45 \lambda^{5}+65 \lambda^{4}-55 \lambda^{3}+28 \lambda^{2}-8 \lambda+1\right)}{10 \lambda^{2}(\lambda-1)^{2}\left(15 \lambda^{2}-15 \lambda+4\right)\left(30 \lambda^{6}-90 \lambda^{5}+110 \lambda^{4}-70 \lambda^{3}+27 \lambda^{2}-7 \lambda+1\right)}\right] \frac{h^{3}}{3 !} W\left(f^{(6)} ; \frac{1}{m}\right)
$$

$\left|S_{\lambda}^{\prime}\left(x_{i+\lambda}\right)-f^{\prime}\left(x_{i+\lambda}\right)\right| \leq \frac{(4 \lambda-2)\left(2 \lambda^{2}-2 \lambda+1\right)\left(5 \lambda^{2}-5 \lambda+1\right)\left(6 \lambda^{4}-12 \lambda^{3}+15 \lambda^{2}-9 \lambda+2\right)}{6\left(15 \lambda^{2}-15 \lambda+4\right)\left(30 \lambda^{6}-90 \lambda^{5}+110 \lambda^{4}-70 \lambda^{3}+27 \lambda^{2}-7 \lambda+1\right)} \frac{h^{5}}{5 !} W\left(f^{(6)} ; \frac{1}{m}\right)$

$\left|S_{n}^{(6)}\left(x_{i}\right)\right| \leq \frac{4(\lambda+1)\left(15 \lambda^{3}-4 \lambda+1\right)}{\lambda^{2}\left(15 \lambda^{2}-15 \lambda+4\right)} h W\left(f^{(6)} ; \frac{1}{m}\right)$

and

$\left|S_{n}^{(6)}\left(x_{i+1}\right)\right| \leq \frac{2 \lambda(\lambda+1)\left(15 \lambda^{2}-7\right)}{\left(15 \lambda^{2}-15 \lambda+4\right)(\lambda-1)^{2}} h W\left(f^{(6)} ; \frac{1}{m}\right)$

\section{Proof:}

From (5),(6) and (8) we have:

$$
\begin{aligned}
h^{5} S_{\lambda}^{(5)}\left(x_{i}\right)= & f\left(x_{i}\right) A_{0}^{(5)}(0)+f\left(x_{i+\lambda}\right) A_{1}^{(5)}(0)+f\left(x_{i+1}\right) A_{2}^{(5)}(0)+h S_{\lambda}^{\prime}\left(x_{i}\right) A_{3}^{(5)}(0) \\
& +h S_{\lambda}^{\prime}\left(x_{i+1}\right) A_{4}^{(5)}(0)+h^{2} f^{\prime \prime}\left(x_{i+\lambda}\right) A_{5}^{(5)}(0)+h^{5} f^{(5)}\left(x_{i+\lambda}\right) A_{6}^{(5)}(0)
\end{aligned}
$$

Hence

$$
\begin{gathered}
h^{5}\left(S_{\lambda}^{(5)}\left(x_{i}\right)-f^{(5)}\left(x_{i}\right)\right)=f\left(x_{i}\right) A_{0}^{(5)}(0)+f\left(x_{i+\lambda}\right) A_{1}^{(5)}(0)+f\left(x_{i+1}\right) A_{2}^{(5)}(0)+h A_{3}^{(5)}(0)\left[S_{\lambda}^{\prime}\left(x_{i}\right)-\right. \\
\left.f^{\prime}\left(x_{i}\right)\right]+ \\
h A_{4}^{(5)}(0)\left[S_{\lambda}^{\prime}\left(x_{i+1}\right)-f^{\prime}\left(x_{i+1}\right)\right]+h^{2} f^{\prime \prime}\left(x_{i+\lambda}\right) A_{5}^{(5)}(0)+h^{5} f^{(5)}\left(x_{i+\lambda}\right) A_{6}^{(5)}(0)-h^{5} f^{(5)}\left(x_{i}\right)+ \\
h A_{3}^{(5)}(0) f^{\prime}\left(x_{i}\right)+h A_{4}^{(5)}(0) f^{\prime}\left(x_{i+1}\right) \\
=\left[\frac{\lambda^{6} h^{6}}{6 !} f^{(6)}\left(\theta_{4, i}\right) A_{1}^{(5)}(0)+\frac{h^{6}}{6 !} f^{(6)}\left(\theta_{2, i}\right) A_{2}^{(5)}(0)+\frac{\lambda^{4} h^{4}}{4 !} f^{(6)}\left(\theta_{6, i}\right) h^{2} A_{5}^{(5)}(0)+\lambda h f^{(6)}\left(\theta_{6, i}\right) h^{5} A_{6}^{(5)}(0)+\right. \\
\left.\frac{h^{5}}{5 !} f^{(6)}\left(\theta_{10, i}\right) h A_{4}^{(5)}(0)\right]+h A_{3}^{(5)}(0)\left[S_{\lambda}^{\prime}\left(x_{i}\right)-f^{\prime}\left(x_{i}\right)\right]+h A_{4}^{(5)}(0)\left[S_{\lambda}^{\prime}\left(x_{i+1}\right)-f^{\prime}\left(x_{i+1}\right)\right] \\
\leq h^{6}\left[\frac{\lambda^{6}}{6 !} A_{1}^{(5)}(0)+\frac{1}{6 !} A_{2}^{(5)}(0)+\frac{\lambda^{4}}{4 !} A_{5}^{(5)}(0)+\lambda A_{6}^{(5)}(0)+\frac{1}{5 !} A_{4}^{(5)}(0)\right] W\left(f^{(6)} ; \frac{1}{m}\right) \\
+\left[A_{3}^{(5)}(0)+A_{4}^{(5)}(0)\right] \frac{2(2 \lambda-1)\left(2 \lambda^{2}-2 \lambda+1\right)\left(5 \lambda^{2}-5 \lambda+1\right)}{\left(30 \lambda^{6}-90 \lambda^{5}+110 \lambda^{4}-70 \lambda^{3}+27 \lambda^{2}-7 \lambda+1\right)} \frac{h^{6}}{5 !} W\left(f^{(6)} ; \frac{1}{m}\right) \\
=\frac{1440(2 \lambda-1)^{2}\left(2 \lambda^{2}-2 \lambda+1\right)\left(3 \lambda^{2}-3 \lambda+1\right)\left(5 \lambda^{2}-5 \lambda+1\right)}{\lambda(\lambda-1)^{2}\left(15 \lambda^{2}-15 \lambda+4\right)\left(30 \lambda^{6}-90 \lambda^{5}+110 \lambda^{4}-70 \lambda^{3}+27 \lambda^{2}-7 \lambda+1\right)} \frac{h^{6}}{5 !} \propto_{2} W\left(f^{(6)} ; \frac{1}{m}\right)
\end{gathered}
$$

where $\left|\propto_{2}\right| \leq 1$ 
By using (12),we get (13).The proofs of (14)-(20) are similar , and we only mention that

$$
\begin{aligned}
& h^{5} S_{\lambda}^{(5)}\left(x_{i+1}\right)=f\left(x_{i}\right) A_{0}^{(5)}(1)+f\left(x_{i+\lambda}\right) A_{1}^{(5)}(1)+f\left(x_{i+1}\right) A_{2}^{(5)}(1)+h S_{\lambda}^{\prime}\left(x_{i}\right) A_{3}^{(5)}(1)+ \\
& h S_{\lambda}^{\prime}\left(x_{i+1}\right) A_{4}^{(5)}(1)+h^{2} f^{\prime \prime}\left(x_{i+\lambda}\right) A_{5}^{(5)}(1)+h^{5} f^{(5)}\left(x_{i+\lambda}\right) A_{6}^{(5)}(1) \text {, } \\
& h^{5} S_{\lambda}^{(5)}\left(x_{i+\lambda}\right)=f\left(x_{i}\right) A_{0}^{(5)}(\lambda)+f\left(x_{i+\lambda}\right) A_{1}^{(5)}(\lambda)+f\left(x_{i+1}\right) A_{2}^{(5)}(\lambda)+h S_{\lambda}^{\prime}\left(x_{i}\right) A_{3}^{(5)}(\lambda)+ \\
& h S_{\lambda}^{\prime}\left(x_{i+1}\right) A_{4}^{(5)}(\lambda)+h^{2} f^{\prime \prime}\left(x_{i+\lambda}\right) A_{5}^{(5)}(\lambda)+h^{5} f^{(5)}\left(x_{i+\lambda}\right) A_{6}^{(5)}(\lambda) \text {, } \\
& h^{4} S_{\lambda}^{(4)}\left(x_{i+\lambda}\right)=f\left(x_{i}\right) A_{0}^{(4)}(\lambda)+f\left(x_{i+\lambda}\right) A_{1}^{(4)}(\lambda)+f\left(x_{i+1}\right) A_{2}^{(4)}(\lambda)+h S_{\lambda}^{\prime}\left(x_{i}\right) A_{3}^{(4)}(\lambda)+ \\
& h S_{\lambda}^{\prime}\left(x_{i+1}\right) A_{4}^{(4)}(\lambda)+h^{2} f^{\prime \prime}\left(x_{i+\lambda}\right) A_{5}^{(4)}(\lambda)+h^{5} f^{(5)}\left(x_{i+\lambda}\right) A_{6}^{(4)}(\lambda) \text {, } \\
& h^{3} S_{\lambda}^{(3)}\left(x_{i+\lambda}\right)=f\left(x_{i}\right) A_{0}^{(3)}(\lambda)+f\left(x_{i+\lambda}\right) A_{1}^{(3)}(\lambda)+f\left(x_{i+1}\right) A_{2}^{(3)}(\lambda)+h S_{\lambda}^{\prime}\left(x_{i}\right) A_{3}^{(3)}(\lambda)+ \\
& h S_{\lambda}^{\prime}\left(x_{i+1}\right) A_{4}^{(3)}(\lambda)+h^{2} f^{\prime \prime}\left(x_{i+\lambda}\right) A_{5}^{(3)}(\lambda)+h^{5} f^{(5)}\left(x_{i+\lambda}\right) A_{6}^{(3)}(\lambda) \text {, } \\
& h S_{\lambda}^{\prime}\left(x_{i+\lambda}\right)=f\left(x_{i}\right) A_{0}^{\prime}(\lambda)+f\left(x_{i+\lambda}\right) A_{1}^{\prime}(\lambda)+f\left(x_{i+1}\right) A_{2}^{\prime}(\lambda)+h S_{\lambda}^{\prime}\left(x_{i}\right) A_{3}^{\prime}(\lambda)+h S_{\lambda}^{\prime}\left(x_{i+1}\right) A_{4}^{\prime}(\lambda) \\
& +h^{2} f^{\prime \prime}\left(x_{i+\lambda}\right) A_{5}^{\prime}(\lambda)+h^{5} f^{(5)}\left(x_{i+\lambda}\right) A_{6}^{\prime}(\lambda) \text {, } \\
& h^{6} S_{\lambda}^{(6)}\left(x_{i}\right)=f\left(x_{i}\right) A_{0}^{(6)}(0)+f\left(x_{i+\lambda}\right) A_{1}^{(6)}(0)+f\left(x_{i+1}\right) A_{2}^{(6)}(0)+h S_{\lambda}^{\prime}\left(x_{i}\right) A_{3}^{(6)}(0)+ \\
& h S_{\lambda}^{\prime}\left(x_{i+1}\right) A_{4}^{(6)}(0)+h^{2} f^{\prime \prime}\left(x_{i+\lambda}\right) A_{5}^{(6)}(0)+h^{5} f^{(5)}\left(x_{i+\lambda}\right) A_{6}^{(6)}(0) \text {, } \\
& h^{6} S_{\lambda}^{(6)}\left(x_{i+1}\right)=f\left(x_{i}\right) A_{0}^{(6)}(1)+f\left(x_{i+\lambda}\right) A_{1}^{(6)}(1)+f\left(x_{i+1}\right) A_{2}^{(6)}(1)+h S_{\lambda}^{\prime}\left(x_{i}\right) A_{3}^{(6)}(1)+ \\
& h S_{\lambda}^{\prime}\left(x_{i+1}\right) A_{4}^{(6)}(1)+h^{2} f^{\prime \prime}\left(x_{i+\lambda}\right) A_{5}^{(6)}(1)+h^{5} f^{(5)}\left(x_{i+\lambda}\right) A_{6}^{(6)}(1) \text {, }
\end{aligned}
$$




\section{Proof of the Theorem 2:}

For $0 \leq y \leq 1$, we obtain

$$
A_{0}(y)+A_{1}(y)+A_{2}(y)=1 \text {. }
$$

Let $x_{i} \leq x \leq x_{i+1}$, on using (21) and (8) we get

$$
\begin{gathered}
S_{n}^{(5)}(x)-f^{(5)}(x)=\left(S_{n}{ }^{(5)}\left(x_{i}\right)-f^{(5)}(x)\right) A_{\circ}\left(\frac{x-x_{i}}{h}\right)+\left(S_{n}{ }^{(5)}\left(x_{i+\lambda}\right)-f^{(5)}(x)\right) A_{1}\left(\frac{x-x_{i}}{h}\right)+ \\
\left(S_{n}^{(5)}\left(x_{i+1}\right)-f^{(5)}(x)\right) A_{2}\left(\frac{x-x_{i}}{h}\right)+h S_{n}{ }^{(6)}\left(x_{i}\right) A_{3}\left(\frac{x-x_{i}}{h}\right)+h S_{n}{ }^{(6)}\left(x_{i+1}\right) A_{4}\left(\frac{x-x_{i}}{h}\right) \\
=E_{1}+E_{2}+E_{3}+E_{4}+E_{5}
\end{gathered}
$$

From (4) it follows that:

$$
\begin{gathered}
E_{1}=\left(S_{n}^{(5)}\left(x_{i}\right)-f^{(5)}(x)\right) A_{0}\left(\frac{x-x_{i}}{h}\right)=\left(S_{n}^{(5)}\left(x_{i}\right)-f^{(5)}\left(x_{i}\right)-\left(x-x_{i}\right) f^{(6)}\left(\theta_{11, i}\right)\right) A_{0}\left(\frac{x-x_{i}}{h}\right) \\
\left|A_{\circ}(x)\right| \leq 1,\left|A_{1}(x)\right| \leq 1, \quad \text { and }\left|A_{2}(x)\right| \leq 1, \quad \text { on } \quad 0 \leq x \leq 1,
\end{gathered}
$$

Since $f^{(5)}(x)=f^{(5)}\left(x_{i}\right)+\left(x-x_{i}\right) f^{(6)}\left(\theta_{11 . i}\right), \quad$ Where $x_{i} \leq \theta_{11, i} \leq x_{i+1}$.

Therefore, on using (13) and $\left|x-x_{i}\right| \leq h$. We obtain

$\left|E_{1}\right| \leq\left[\frac{12(2 \lambda-1)^{2}\left(2 \lambda^{2}-2 \lambda+1\right)\left(3 \lambda^{2}-3 \lambda+1\right)\left(5 \lambda^{2}-5 \lambda+1\right)}{\lambda(\lambda-1)^{2}\left(15 \lambda^{2}-15 \lambda+4\right)\left(30 \lambda^{6}-90 \lambda^{5}+110 \lambda^{4}-70 \lambda^{3}+27 \lambda^{2}-7 \lambda+1\right)}+1\right] h W\left(f^{(6)} ; \frac{1}{m}\right)$

(23) Similarly,

$$
\begin{aligned}
& E_{3}=\left[S_{n}^{(5)}\left(x_{i+1}\right)-f^{(5)}(x)\right] A_{2}\left(\frac{x-x_{i}}{h}\right) \\
& =\left[S_{n}^{(5)}\left(x_{i+1}\right)-f^{(5)}\left(x_{i}\right)-\left(x-x_{i}\right) f^{(6)}\left(\theta_{11, i}\right)\right] A_{2}\left(\frac{x-x_{i}}{h}\right) \\
& \left|E_{3}\right| \leq\left[\frac{2(2 \lambda-1)^{2}\left(2 \lambda^{2}-2 \lambda+1\right)\left(3 \lambda^{2}-3 \lambda+1\right)\left(5 \lambda^{2}-5 \lambda+1\right)}{\lambda^{2}(\lambda-1)\left(15 \lambda^{2}-15 \lambda+4\right)\left(30 \lambda^{6}-90 \lambda^{5}+110 \lambda^{4}-70 \lambda^{3}+27 \lambda^{2}-7 \lambda+1\right)}+1\right] h W\left(f^{(6)} ; \frac{1}{m}\right)
\end{aligned}
$$


and

$$
\begin{aligned}
E_{2}=\left[S_{n}^{(5)}\left(x_{i+\lambda}\right)\right. & \left.-f^{(5)}(x)\right] A_{1}\left(\frac{x-x_{i}}{h}\right) \\
= & {\left[S_{n}^{(5)}\left(x_{i+\lambda}\right)-f^{(5)}\left(x_{i}\right)-\left(x-x_{i}\right) f^{(6)}\left(\theta_{11, i}\right)\right] A_{1}\left(\frac{x-x_{i}}{h}\right) }
\end{aligned}
$$

$\left|E_{2}\right| \leq h(\lambda+1) W\left(f^{(6)} ; \frac{1}{m}\right)$

$E_{4}=h S_{\lambda}^{(6)}\left(x_{i}\right) A_{3}(t)$

$\left|E_{4}\right| \leq \frac{4(\lambda+1)\left(15 \lambda^{3}-4 \lambda+1\right)}{\lambda^{2}\left(15 \lambda^{2}-15 \lambda+4\right)} h W\left(f^{(6)} ; \frac{1}{m}\right)$

$E_{5}=h S_{\lambda}^{(6)}\left(x_{i+1}\right) A_{4}(t)$.

$\left|E_{5}\right| \leq \frac{2 \lambda(\lambda+1)\left(15 \lambda^{2}-7\right)}{\left(15 \lambda^{2}-15 \lambda+4\right)(\lambda-1)^{2}} h W\left(f^{(6)} ; \frac{1}{m}\right)$

Therefore by using (23)-(27) and putting in (22) we obtain

$$
\begin{aligned}
&\left|S_{\lambda}^{(5)}(x)-f^{(5)}(x)\right| \leq \frac{1}{\lambda^{2}\left(15 \lambda^{2}-15 \lambda+4\right)\left(30 \lambda^{6}-90 \lambda^{5}+110 \lambda^{4}-70 \lambda^{3}+27 \lambda^{2}-7 \lambda+1\right)(\lambda-1)^{2}}\left[450 \lambda^{13}+\right. \\
& 1350 \lambda^{12}-9930 \lambda^{11}+20310 \lambda^{10}-20005 \lambda^{9}+7855 \lambda^{8}+4419 \lambda^{7}-8626 \lambda^{6}+ \\
&\left.6459 \lambda^{5}-2935 \lambda^{4}+795 \lambda^{3}-94 \lambda^{2}-6 \lambda+2\right] h W\left(f^{(6)} ; \frac{1}{m}\right)
\end{aligned}
$$

This proves Theorem 2 for $\mathrm{r}=5$. To Prove the Theorem 2 for $\mathrm{r}=4$ :

and using (16) and (28) we obtain

since

$$
\begin{aligned}
& S_{n}^{(4)}(x)- f^{(4)}(x)=\int_{x_{i+\lambda}}^{x}\left(S_{n}^{(5)}(t)-f^{(5)}(t)\right) d t+S_{n}^{(4)}\left(x_{i+\lambda}\right)-f^{(4)}\left(x_{i+\lambda}\right) . \\
&\left|S^{(4)}(x)-f^{(4)}(x)\right| \leq \frac{h^{2}}{10 \lambda^{2}\left(15 \lambda^{2}-15 \lambda+4\right)\left(30 \lambda^{6}-90 \lambda^{5}+110 \lambda^{4}-70 \lambda^{3}+27 \lambda^{2}-7 \lambda+1\right)(\lambda-1)^{2}} \\
&\left(2250 \lambda^{13}+6750 \lambda^{12}-49650 \lambda^{11}+101950 \lambda^{10}-102025 \lambda^{9}+44055 \lambda^{8}+\right. \\
&\left.14975 \lambda^{7}-35880 \lambda^{6}+27065 \lambda^{5}-11989 \lambda^{4}+3013 \lambda^{3}-242 \lambda^{2}-62 \lambda+12\right) \\
& W\left(f^{(6)} ; \frac{1}{m}\right)
\end{aligned}
$$


which proves Theorem 2 for $r=4$. To Prove the Theorem 2 for $r=3$ :

and using (17) and (29) we obtain

Since

$$
\begin{aligned}
S_{n}^{(3)}(x)-f^{(3)}(x) & =\int_{x_{i+\lambda}}^{x}\left(S_{n}^{(4)}(t)-f^{(4)}(t)\right) d t+S_{n}^{(3)}\left(x_{i+\lambda}\right)-f^{(3)}\left(x_{i+\lambda}\right) . \\
\left|S^{(3)}(x)-f^{(3)}(x)\right| \leq & \frac{h^{3}}{30 \lambda^{2}\left(15 \lambda^{2}-15 \lambda+4\right)\left(30 \lambda^{6}-90 \lambda^{5}+110 \lambda^{4}-70 \lambda^{3}+27 \lambda^{2}-7 \lambda+1\right)(\lambda-1)^{2}}\left(6750 \lambda^{14}-\right. \\
& 38250 \lambda^{13}+114300 \lambda^{12}-215850 \lambda^{11}+267325 \lambda^{10}-212265 \lambda^{9}+ \\
& 92355 \lambda^{8}+3990 \lambda^{7}-38075 \lambda^{6}+30567 \lambda^{5}-13839 \lambda^{4}+3622 \lambda^{3}- \\
& \left.373 \lambda^{2}-45 \lambda+11\right) W\left(f^{(6)} ; \frac{1}{m}\right)
\end{aligned}
$$

Which proves Theorem 2 for $\mathrm{r}=3$. To prove Theorem 2 for $\mathrm{r}=2$,

Since $S_{n}^{(2)}\left(x_{i+\lambda}\right)-f^{(2)}\left(x_{i+\lambda}\right)=0$, and using (30)

$$
\begin{aligned}
& S_{n}^{\prime \prime}(x)- f^{\prime \prime}(x)=\int_{x_{i+\lambda}}^{x}\left(S_{n}^{(3)}(t)-f^{(3)}(t)\right) d t+S_{n}^{\prime \prime}\left(x_{i+\lambda}\right)-f^{\prime \prime}\left(x_{i+\lambda}\right) . \\
&\left|S^{(2)}(x)-f^{(2)}(x)\right| \leq \frac{h^{4}}{120 \lambda^{2}\left(15 \lambda^{2}-15 \lambda+4\right)\left(30 \lambda^{6}-90 \lambda^{5}+110 \lambda^{4}-70 \lambda^{3}+27 \lambda^{2}-7 \lambda+1\right)(\lambda-1)^{2}}\left(6750 \lambda^{14}-\right. \\
& \quad 38250 \lambda^{13}+114300 \lambda^{12}-215850 \lambda^{11}+267325 \lambda^{10}-212265 \lambda^{9}+92355 \lambda^{8}+ \\
&\left.\quad 3990 \lambda^{7}-38075 \lambda^{6}+30567 \lambda^{5}-13839 \lambda^{4}+3622 \lambda^{3}-373 \lambda^{2}-45 \lambda+11\right) \\
& \quad W\left(f^{(6)} ; \frac{1}{m}\right)
\end{aligned}
$$

Which proves Theorem 2 for $\mathrm{r}=2$. To prove Theorem 2 for $\mathrm{r}=1$,

On using (18) and (31) we obtain

since

$$
S_{n}^{\prime}(x)-f^{\prime}(x)=\int_{x_{i+\lambda}}^{x}\left(S_{n}^{(2)}(t)-f^{(2)}(t)\right) d t+S_{n}^{\prime}\left(x_{i+\lambda}\right)-f^{\prime}\left(x_{i+\lambda}\right) .
$$




$$
\begin{aligned}
\left|S^{\prime}(x)-f^{\prime}(x)\right| \leq & \frac{h^{5}}{1800 \lambda^{2}\left(15 \lambda^{2}-15 \lambda+4\right)\left(30 \lambda^{6}-90 \lambda^{5}+110 \lambda^{4}-70 \lambda^{3}+27 \lambda^{2}-7 \lambda+1\right)(\lambda-1)^{2}}\left(20250 \lambda^{14}-\right. \\
& 114150 \lambda^{13}+339000 \lambda^{12}-635430 \lambda^{11}+778215 \lambda^{10}-604405 \lambda^{9}+ \\
& 245160 \lambda^{8}+34920 \lambda^{7}-126180 \lambda^{6}+96086 \lambda^{5}-42587 \lambda^{4}+11021 \lambda^{3}- \\
& \left.1129 \lambda^{2}-135 \lambda+33\right) W\left(f^{(6)} ; \frac{1}{m}\right)
\end{aligned}
$$

Which proves Theorem 2 for $\mathrm{r}=1$. To prove Theorem 2 for $\mathrm{r}=0$,

$$
\begin{aligned}
& \text { since } S_{n}(x)-f(x)=\int_{x_{i+\lambda}}^{x}\left(S_{n}^{\prime}(t)-f^{\prime}(t)\right) d t+S_{n}\left(x_{i+\lambda}\right)-f\left(x_{i+\lambda}\right) \\
& S_{n}\left(x_{i+\lambda}\right)-f\left(x_{i+\lambda}\right)=0 \text {,and using (32) } \\
& \qquad S(x)-f(x) \mid \leq \frac{h^{6}}{10800 \lambda^{2}\left(15 \lambda^{2}-15 \lambda+4\right)\left(30 \lambda^{6}-90 \lambda^{5}+110 \lambda^{4}-70 \lambda^{3}+27 \lambda^{2}-7 \lambda+1\right)(\lambda-1)^{2}} \\
& \left(20250 \lambda^{14}-114150 \lambda^{13}+339000 \lambda^{12}-635430 \lambda^{11}+778215 \lambda^{10}-604405 \lambda^{9}+\right. \\
& 245160 \lambda^{8}+34920 \lambda^{7}-126180 \lambda^{6}+96086 \lambda^{5}-42587 \lambda^{4}+11021 \lambda^{3}-1129 \lambda^{2}- \\
& 135 \lambda+33) W\left(f^{(6)} ; \frac{1}{m}\right)
\end{aligned}
$$

\section{Conclusion:}

These generalize are prefer to interpolation by sixtic spline on uniform meshes to the use $(0,2,5)$. We also can use this idea to generalize for different Lacunary type for example $(0,1,3)$, $(0,2,3), \ldots$ etc.

\section{References:}

[1] Saeed, R.K. and Jwamer, K.H., (2005), Minimizing error bounds in Lacunary interpolation by spline function, $(0,1,4)$ case. Journal of Al. Nahrain University, 8 (2), pp.114-119.

[2] Kanth, A.S., V.R. and V. Bhattacharya, 2006. Cubic spline for a class on non-linear singular boundary value problems arising in physiology. Applied Math. Comput., 174: 768-774.

[3] Jwamer, K.H., (2007), Minimizing error bounds in (0,2,3) Lacunary interpolation by sixtic spline function, Journal of Mathematics and Statistics, USA, 3(4),pp.249-256 .

[4] Jwamer, K.H. and Ghafoor,R.K.,(2010):Generalization of (0,4) Lacunary Interpolation by Quantic Spline, Journal of Mathematics and Statistics in USA 6(1):72-78.

[5] Jwamer, K.H. and Ghafoor,R.K (2011): New Construction and New Error Bounds For $(0,2,4)$ Lacunary Interpolation By Six Degree Spline, Mosel university, Al-Rafidain journal of computer Sciences and Mathematics,Vol.(8) No.(1). 